\title{
Virtual Commissioning, Digital Twin and Control Strategies Applied in the Industrial Robot PUMA 560
}

\author{
Rogério Adas Pereira Vitalli \\ Department of Robotics Engineering, Robotics Advanced Institute (IAR), São Paulo, Brazil
}

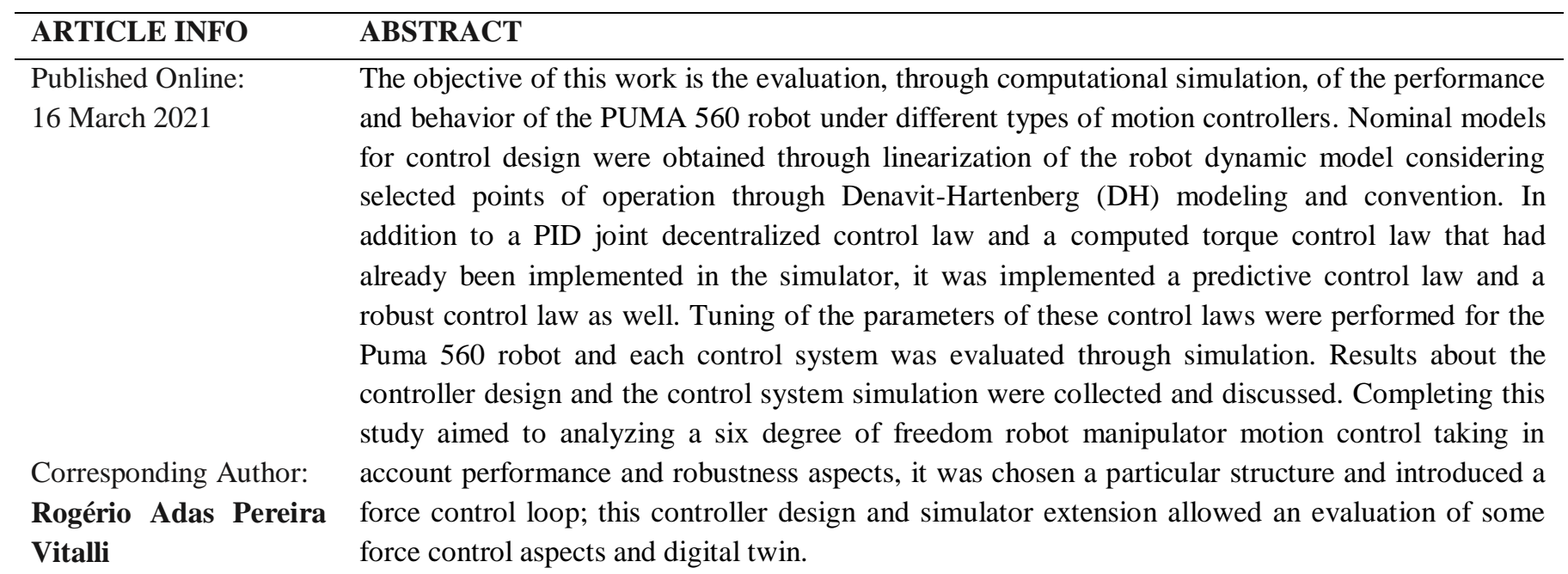

KEYWORDS: PUMA 560, Denavit-Hartenberg (DH), Digital Twin, Roboticist

\section{INTRODUCTION}

In mid 1945, Professor Denavit, together with his mentor Hartenberg, developed a notation in which the objective was purely to seek ease in the design of warlike mechanisms. At that time, attention was focused on the interests of World War II. Two decades later, this notation caught the attention of researcher R. P. PAUL, who was studying orientation problems in rigid bodies in M.I.T. [9]. In the study of Robotics, there is a constant interest in the three dimensional location of objects, such as the links of the manipulator, parts, tools and others, in the work environment of the manipulator. In order to simultaneously describe the position and orientation of a body in space (6 GDL required), a coordinated system will be fixed to the object, thereby allowing the position and orientation of this system (and therefore of the object) to be described with relation to a coordinated reference system. [9], [10], [7].

Around the 1960s, when the first industrial robots were launched, their price was very high and only very few companies located in developed countries had access to them. Today the reality is another. Industrial automation was and is a major driver of robotics technology. Each time it is concerned with improving the devices, providing them with "intelligence" to perform the necessary tasks. Corke P. (2007), points out that the advantages resulting from the use of robots are numerous. Among them, we highlight the increase in productivity, the improvement and consistency in the final quality of the product. In practice, the application of robots in the industry requires a reliable and robust solution that consistently performs the predetermined functions.

The design of robotic manipulators and their control systems presents many difficulties that arise from the complexity of their dynamic models. As a result, one of the resources that assist in the development of projects are the "simulation tools" that can assess, with greater comprehensiveness, the behavior and performance of the control systems, perform tuning of controllers and test different solutions "offline".

Rocha, Tornetto and Dias (2011) developed a general robotic simulator in a Matlab / Simulink environment. In a very efficient and generalized way, the simulator computes the direct dynamics of a generic, rigid-link, generic robotic manipulator. Other functional blocks are added in order to guarantee amplitude and efficiency in the simulation of the global system. In Atique et al (2018), the simulator was 
extended to simulate force / torque control. This simulator will serve as a basis for the work developed here.

This work focuses on the evaluation, with computer simulation, of the performance of the PUMA 560 robot under different types of motion controllers. The nominal models for the control project were obtained with the linearized dynamic model of the robot that considers selected operating points.

\section{MODELING AND DENAVIT-HARTENBERG (DH) CONVENTION}

The Denavit-Hartenberg (DH) notation is introduced as a systematic method for describing a link separating a successive pair of joints (rotating or prismatic) and the kinematic relationship between a pair of adjacent links (Denavit, J. and Hartenberg R. Scheunemann, 1955). The base of the manipulator is considered as link 0 and the other links receive an increasing number from 1 to $n$, progressively from the base to the terminal organ. Thus, a minimum number of parameters is used in the description and shown in figure 1 :

$a_{i}$ - length of the common normal $\left(\mathrm{H}_{i} \mathrm{O}_{i}\right)$, that is, the distance between the axis of the joints at each end of link $i$, measured along the line normal to both axes along $\left(\hat{x}_{i}\right)$;

$d_{i}$ - length between the origin $\left(O_{i-1}\right)$ and the point $\left(H_{i}\right)$, that is, the distance between the common normal, $\left(H_{i-1} O_{i-1}\right)$ and $\left(H_{i} O_{i}\right)$, measured along $\left(\hat{z}_{i-1}\right)$;

$\theta_{i}$ - angle between the axis $\left(\hat{x}_{i-1}\right)$ and the normal common angle axis $\left(\hat{x}_{i}\right)$, measured around the axis $\left(\hat{z}_{i-1}\right)$ in the direction of the right hand;

$\alpha_{i}$ - angle between the axis of joint i $\left(\hat{z}_{i-1}\right)$ and the axis of joint $\mathrm{i}+1\left(z_{i}\right)$, in the direction of the right hand, measured around $\left(\hat{x}_{i}\right)$.

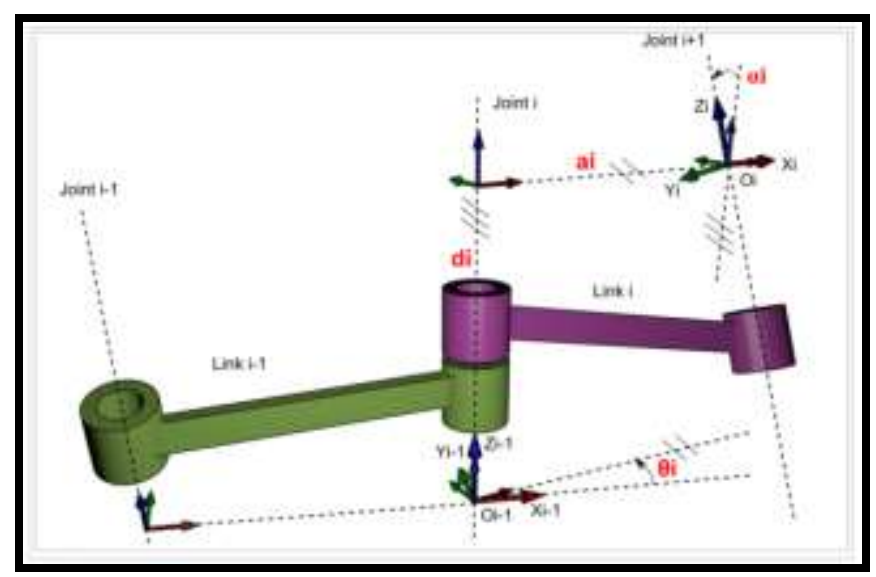

Fig 1: Denavit-Hartenberg (DH) notation

The parameters (link length) and (torsion angle) are constant and determined by the link geometry. Only one of the two other parameters, (length between common normals) and (angle of rotation), varies when joint i moves: when joint $i$ is prismatic and when joint $i$ is rotating; this variable is generally called the joint variable.

\section{A. Industrial Robot PUMA 560}

It is important that the robotic manipulator is always in its home position (with the links at $0^{\circ}$ or $90^{\circ}$ from each other) to facilitate the convention of the D-H parameters and the possibility of creating a reliable algorithm. According to (Denavit, J. and Hartenberg R. Scheunemann, 1955), a more systematic and structured methodology determines that the joints of the robot must be numbered in ascending order, starting at the base of the manipulator and ending with the last link, associated with the terminal organ. Following the D-H notation, an outline of the PUMA 560 robotic manipulator of six revolute joints is shown in figure 2 .

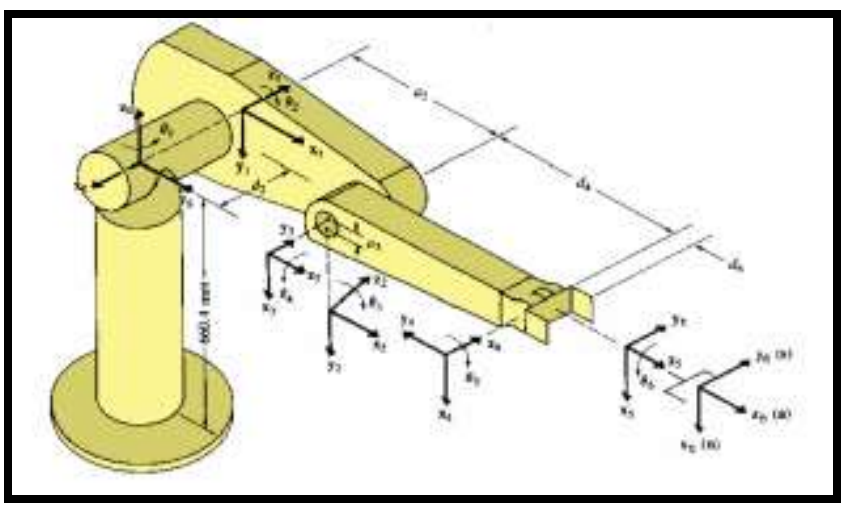

Fig 2: Systematic Method Denavit-Hartenberg ( PUMA $560)$

\section{B. Homogeneous Transformation Matrix}

The D-H matrix designated by the initial $\mathrm{T}$ is considered transformational because it establishes a relationship between Cartesian coordinates and homogeneous coordinates, in addition to joining translation and rotation movements in a single matrix. The local scale factor and / or 3x3 rotation matrix is inserted within the transformation matrix $\mathrm{T}$ and considers the three-dimensional rotations of rigid bodies. This matrix can also be replaced by another of the same $3 \times 3$ dimension, in which it considers the rotations from the Euler angles. When the value of this orientation matrix is identity, we say that the points are collinear. Homogeneous or isometric transformations preserve angles and distances. Recently, the global manufacturers of robots are replacing this matrix with quaternions with the objective of gaining computational time and decreasing singularities (A. Kovalchuk. and Nauka I., 2015). The translation vector is always $3 \times 1$ because it considers the linear movements on the axes, respectively. The last line of the D-H matrix was developed by researchers in the computing area in order to control the dynamics of the matrix and it consists of elements of projection and scale factor. The vast majority of perspectives are constituted by the triple [llll 00 that maps orthogonal vectors, that is, lines are visualized; when the 
triple is formed by [llllll, 111 , path points are mapped, which are fundamental for interpolation of trajectories. Finally, the global scale factor or digital zoom is the element responsible for viewing the results; the only value that should not be assigned to it is zero, as the points or lines would tend to infinity $(\mathrm{det}=0)$. If this value is positive, the "object" that we want to visualize is below the observer; if the assumed value is negative, the "object" would be beyond the observer. In other words, the scale factor amplifies or reduces the size of the "object;" in general its value is always 1 which characterizes the real size of the object. [5], [8].

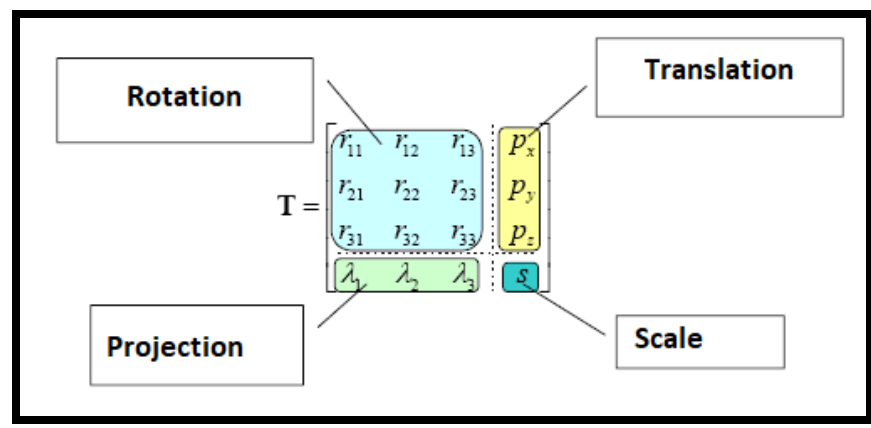

Fig 3: Matrix D-H

${ }_{i}^{i-1} T=\operatorname{ROT}\left(\hat{z}_{i-1}, \theta_{i}\right) \cdot \operatorname{TRANS}\left(\hat{z}_{i-1}, d_{i}\right) \cdot \operatorname{TRANS}\left(\hat{x}_{i}, a_{i}\right) \cdot \operatorname{ROT}\left(\hat{x}_{i}, \alpha_{i}\right)$

$T_{1}^{0}=\left[\begin{array}{cccc}c_{1} & 0 & -s_{1} & 0 \\ s_{1} & 0 & c_{1} & 0 \\ 0 & -1 & 0 & 0 \\ 0 & 0 & 0 & 1\end{array}\right]$

$T_{2}^{1}=\left[\begin{array}{cccc}c_{2} & -s_{2} & 0 & a_{2} \cdot c_{2} \\ s_{2} & c_{2} & 0 & a_{2} \cdot s_{2} \\ 0 & 0 & 1 & d_{2} \\ 0 & 0 & 0 & 1\end{array}\right]$

$T_{3}^{2}=\left[\begin{array}{cccc}c_{3} & 0 & s_{3} & a_{3} \cdot c_{3} \\ s_{3} & 0 & -c_{3} & a_{3} \cdot s_{3} \\ 0 & 1 & 0 & 0 \\ 0 & 0 & 0 & 1\end{array}\right]$

$T_{4}^{3}=\left[\begin{array}{cccc}c_{4} & 0 & -s_{4} & 0 \\ s_{4} & 0 & c_{4} & 0 \\ 0 & -1 & 0 & d_{4} \\ 0 & 0 & 0 & 1\end{array}\right]$

$T_{5}^{4}=\left[\begin{array}{cccc}c_{5} & 0 & s_{5} & 0 \\ s_{5} & 0 & -c_{5} & 0 \\ 0 & 1 & 0 & 0 \\ 0 & 0 & 0 & 1\end{array}\right]$

$$
T_{6}^{5}=\left[\begin{array}{cccc}
c_{6} & -s_{6} & 0 & 0 \\
s_{6} & c_{6} & 0 & 0 \\
0 & 0 & 1 & d_{6} \\
0 & 0 & 0 & 1
\end{array}\right]
$$

In possession of the transformation matrices between the consecutive links (2.2-2.7), the direct kinematics of the manipulator is obtained:

$T_{6}^{0}=T_{1}^{0} \cdot T_{2}^{1} \cdot T_{3}^{2} \cdot T_{4}^{3} \cdot T_{5}^{4} \cdot T_{6}^{5}$

\section{Kinematics Dynamics and Simulation}

For simulating the dynamics of the manipulator, second order models for the actuators were considered. We were able to implement the Newton-Euler formulation, responsible for the general solution of the dynamics of openchain robotic manipulators and rigid bodies. By simply reading a data matrix (kinematic and dynamic parameters of the robot) we are able to solve the direct dynamics of the manipulator.

Typical disturbances to consider are external torques and forces. Another possibility is to consider the variation in mass in the terminal organ, simulating the grasping of a body. The motion path generator tells the controller which path is desired for the manipulator. There are several ways to define these trajectories, such as: cubic spline, 4-3-4, and others. In order to consider typical disturbances, a contact force / torque generator was implemented. With respect to force / torque control, the experimental results found by (Paul, 1981) for different force control laws with varying reference inputs, point out that the law of proportionalintegral control (PI) provides a better performance. The main advantage is the first order admittance, where it is not necessary to require the rate of change of force error. Shown in Table 1. Parameters of the PUMA 560. [3], [7], [10].

\begin{tabular}{|l|l|l|l|l|l|}
\hline Joint $\mathrm{i}$ & $\theta_{\mathrm{i}}$ & $\alpha_{\mathrm{i}}$ & $\mathrm{a}_{\mathrm{i}}$ & $\mathrm{d}_{\mathrm{i}}$ & Axis Range \\
\hline 1 & $90^{\circ}$ & $-90^{\circ}$ & 0 & 0 & $-160^{\circ} \mathrm{a} 160^{\circ}$ \\
\hline 2 & $0^{\circ}$ & $0^{\circ}$ & $\mathrm{a}_{2}$ & $\mathrm{~d}_{2}$ & $-225^{\circ} \mathrm{a} 45^{\circ}$ \\
\hline 3 & $90^{\circ}$ & $90^{\circ}$ & $-\mathrm{a}_{3}$ & 0 & $-45^{\circ} \mathrm{a} 225^{\circ}$ \\
\hline 4 & $0^{\circ}$ & $-90^{\circ}$ & 0 & $\mathrm{~d}_{4}$ & $-110^{\circ} \mathrm{a} 170^{\circ}$ \\
\hline 5 & $0^{\circ}$ & $90^{\circ}$ & 0 & 0 & $-100^{\circ} \mathrm{a} 100^{\circ}$ \\
\hline 6 & $0^{\circ}$ & $0^{\circ}$ & 0 & $\mathrm{~d}_{6}$ & $-266^{\circ}$ a $266^{\circ}$ \\
\hline
\end{tabular}

Data: $\mathrm{a}_{2}=431.8 \mathrm{~mm} ; \mathrm{a}_{3}=20.32 \mathrm{~mm} ; \mathrm{d}_{2}=149.09 \mathrm{~mm}$; $\mathrm{d}_{4}=433.07 \mathrm{~mm} ; \mathrm{d}_{6}=56.25 \mathrm{~mm}$

\section{Virtual Commissioning and Digital Twin}

The biggest benefit of virtual commissioning is savings. You can test an investment in advance, which could be very expensive. It would be interesting to follow your project from the beginning using the digital twin of the mechatronics line: from the concept phase to the development for commissioning and optimization during 
operation. Due to simulation and virtual commissioning, it is possible to reduce the time to market (TTM), reduce error costs, increase quality, and reduce risks during actual commissioning. [6]

The digital twin of a robotic cell allows to parallel the mechanical and electrical design as well as an automation and robotics engineering. Waiting times for phases of information exchange between teams can be avoided in this way. In addition, virtual commissioning can take place in a digital office environment, instead of using expensive prototypes or real machines at the plant's operating site. Through the simulation of the machine, extensive tests allow the detection and correction of errors in the design and functions, making real commissioning very fast. According to the Six Sigmas management system, error costs increase by a factor of ten (10) with each stage of development. With simulation, perceptions of virtual tests can improve engineering quality at an early stage. Testing the original PLC code with virtual controllers during actual commissioning increases confidence that the interaction between the electrical and mechanical parts and the controller is working as intended by the customer. This helps to avoid high error costs. Problems during actual commissioning take time and increase labor and material costs, especially with international projects. To work around problems, everything can be tested safely during virtual commissioning without the need for the customer's robotic cell operator. Industrial robots are designed to work constantly, with no downtime after commissioning. Optimizations or renovations require access to the machine, which is not always possible. Thanks to the digital twin of the real robot, it is possible to validate all actions during operation, this reduces downtime to a minimum. [5], [6].

\section{RESULTS}

The results presented show the performance of the PID, TC, ROB and PRED controllers simulating each of the joints of the PUMA 560 robotic manipulator separately, that is, not coupled. For these figures, no type of mass variation was considered in the robot's wrist.

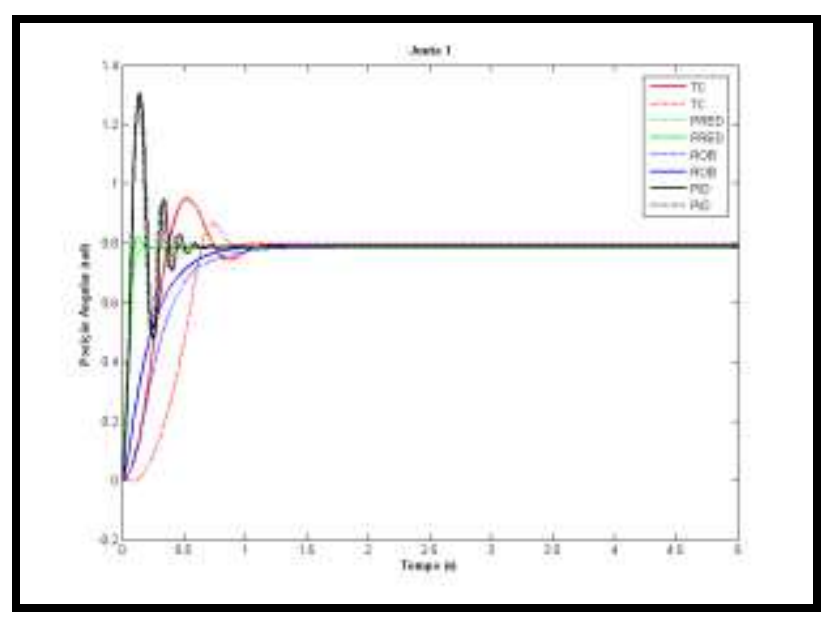

Fig 4: Joint-01 simulation under the action of the four controllers with the presence of coulomb friction, with no change in mass in the last link, with the actual gear ratio of the PUMA 560 (continuous dashed) and with a single or directly connected gear ratio (dashed line)

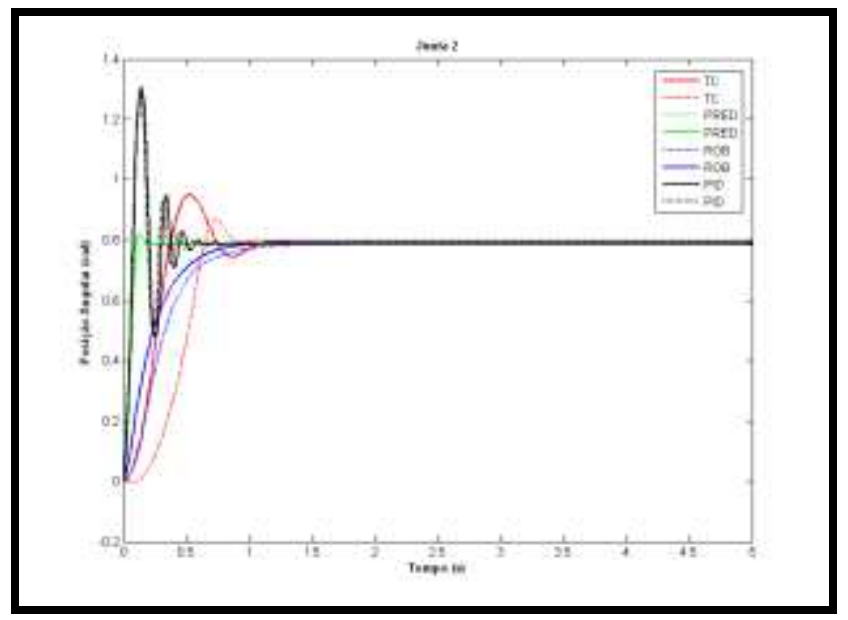

Fig 5: Joint-02 simulation under the action of the four controllers with the presence of coulomb friction, with no change in mass in the last link, with the actual gear ratio of the PUMA 560 (continuous dashed) and with a single or directly connected gear ratio (dashed line)

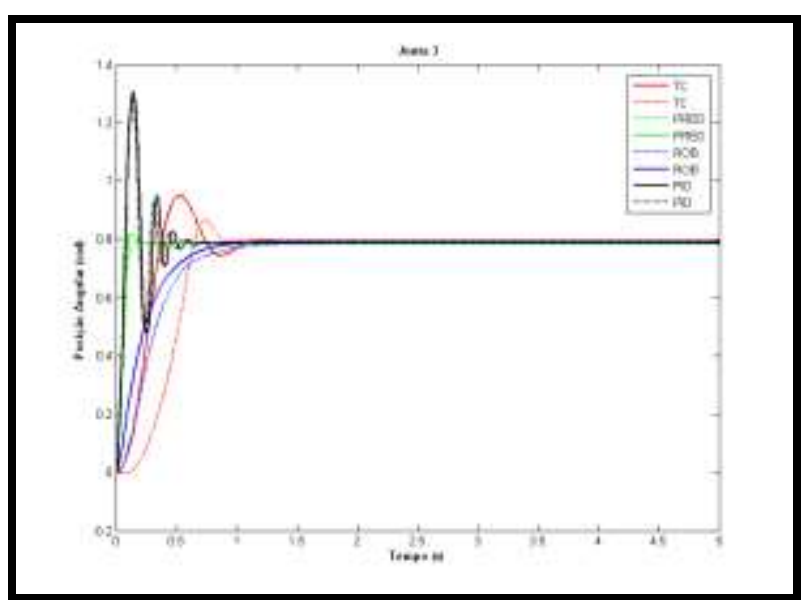

Fig 6: Joint-03 simulation under the action of the four controllers with the presence of coulomb friction, with no change in mass in the last link, with the actual gear ratio of the PUMA 560 (continuous dashed) and with a single or directly connected gear ratio (dashed line)

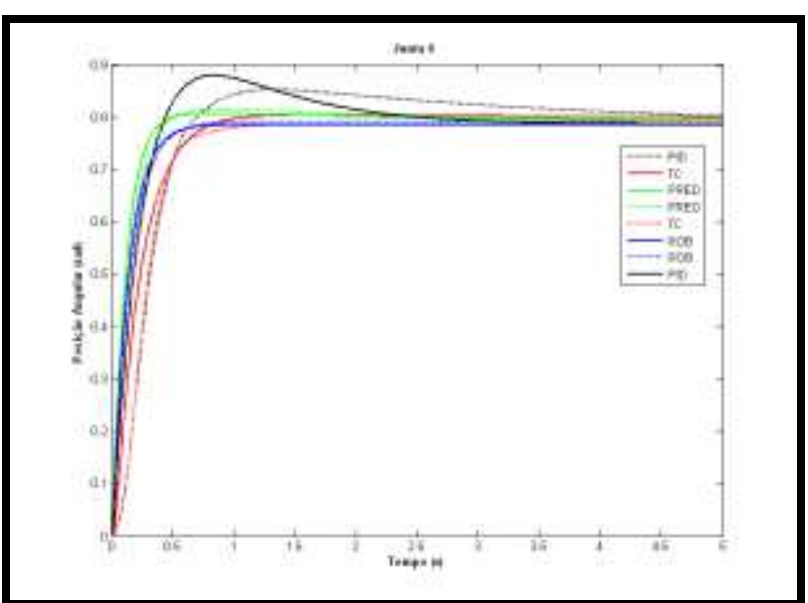

Fig 7: Joint-04 simulation under the action of the four controllers with the presence of coulomb friction, with no 
change in mass in the last link, with the actual gear ratio of the PUMA 560 (continuous dashed) and with a single or directly connected gear ratio (dashed line)

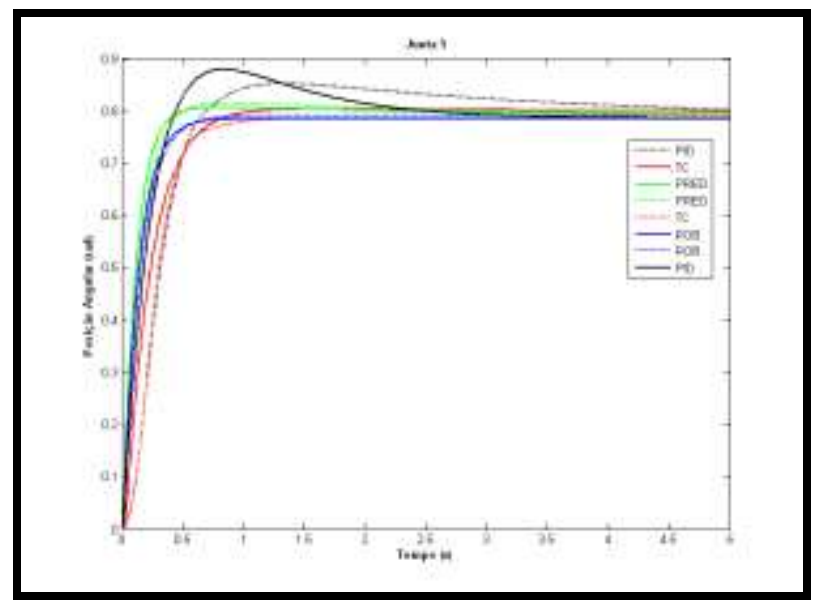

Fig 8: Joint-05 simulation under the action of the four controllers with the presence of coulomb friction, with no change in mass in the last link, with the actual gear ratio of the PUMA 560 (continuous dashed) and with a single or directly connected gear ratio (dashed line)

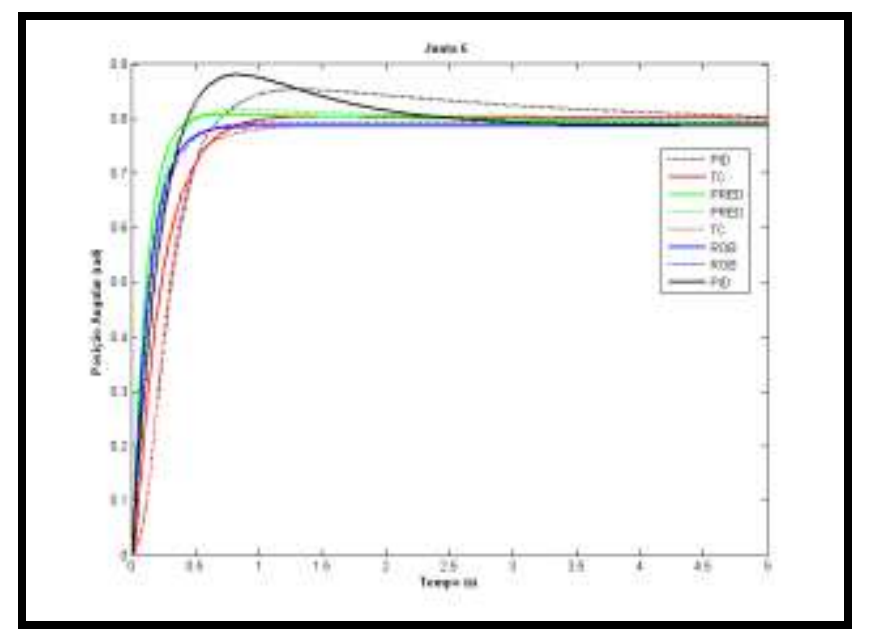

Fig 9: Joint-06 simulation under the action of the four controllers with the presence of coulomb friction, with no change in mass in the last link, with the actual gear ratio of the PUMA 560 (continuous dashed) and with a single or directly connected gear ratio (dashed line)

\section{DISCUSSION AND CONCLUSION}

The results presented by figures 4 to 9 show the performance of the controllers: PID, (Proportional, Integral, Derivative) TC (Computed Torque), ROB (Robust) and PRED (Predictive) simulating each of the joints of the PUMA 560 robotic manipulator. these figures did not consider any type of mass variation in the robot's wrist. It is clearly noted that the joint-06 showed less angular variation in position than the others, as expected due to its low inertia.

The design of the PID controller provided low-order gains and the results provided were not the best performers. Joint-01, with greater inertia, is a classic example, where the stabilization time exceeds four seconds. The TC controller performed better than the previous one, although the base joints $(1,2,3)$ contained a $25 \%$ overshoot that took longer to stabilize than the wrist joints $(4,5,6)$. The ROB controller calculated according to the plant in the state space performed corrective actions for a highly nonlinear dynamics of the PUMA 560, but a little less efficient than the PRED controller. The estimated peak time of two seconds has been satisfied for all wrist joints and can be viewed following the action of the robust controller in a continuous blue line.

Finally, it is possible to state that in the absence of restrictions and without rigid disturbances (no load or tool on the robot's handle), the predictive controller was much better than the others. For comparison purposes, the optimal parameters of the controller (obtained empirically) will be kept the same for all cases analyzed below.

\section{REFERENCES}

1. Atique, Md., Moin Uddin, Sarker, Md. Rafiqul Islam and Ahad, Md. Atiqur Rahman. 2018. Development of an 8DOF quadruped robot and implementation of Inverse Kinematics using Denavit-Hartenberg convention. Heliyon, Vol.4 (12)

2. A. K. Kovalchuk., Nauka I. obrazovanie (Moskva), 2015 Modified Denavit-Hartenberg Coordinate System for Robot Actuating Mechanisms with Tree-like Kinematic Structure. Issue 11, pp.12-30

3. Corke, P.I. 2007. A Simple and Systematic Approach to Assigning Denavit-Hartenberg Parameters. IEEE Transactions on Robotics, Vol.23(3), pp.590-594

4. Denavit, Jacques and Hartenberg R. Scheunemann. 1955. A kinematic notation for lower-pair mechanisms based on matrices, Trans ASME J. Appl. Mech. 23: 215-221

5. Ding, Feng., Liu, Cong. 2018. Applying coordinate fixed Denavit-Hartenberg method to solve the workspace of drilling robot arm. International journal of advanced robotic systems, Vol.15(4)

6. Flanders, Megan., and Kavanagh, Richard. 2015, Build A-Robot: Using virtual reality to visualize the Denavit-Hartenberg parameters. Computer Applications in Engineering Education, Vol.23(6), pp.846-853

7. Hartenberg R. Scheunemann, and Denavit, Jacques, 1965. Kinematic synthesis of linkages, Col: McGraw-Hill series in mechanical engineering. New York: McGraw-Hill. 435 pages

8. Luo Qingsheng and Julpri A. Sinergi. 2018. Analysis of Kinematic for Legs of a Hexapod using Denavit-Hartenberg Convention. Fakultas Teknologi Industri Univeritas Mercu Buana, Vol.22(2), pp.69-76

9. Paul, Richard. 1981. Robot manipulators: mathematics, programming, and control: the 
"Virtual Commissioning, Digital Twin and Control Strategies Applied in the Industrial Robot PUMA 560" computer control of robot manipulators. Cambridge, MA: MIT

10. Rocha C.R., Tonetto, C.P and Dias, A. 2011. A comparison between the Denavit-Hartenberg and the screw-based methods used in kinematic modeling of robot manipulators. Robotics and computer-integrated manufacturing, Vol.27(4), pp.723-728 